Association for Information Systems

AIS Electronic Library (AISeL)

ICEB 2012 Proceedings

International Conference on Electronic Business

(ICEB)

Fall 10-12-2012

Some Concepts and Theorems of Uncertain Random Process

Kai Yao

Jinwu Gao

Follow this and additional works at: https://aisel.aisnet.org/iceb2012

This material is brought to you by the International Conference on Electronic Business (ICEB) at AIS Electronic Library (AISeL). It has been accepted for inclusion in ICEB 2012 Proceedings by an authorized administrator of AIS Electronic Library (AISeL). For more information, please contact elibrary@aisnet.org. 


\title{
Some Concepts and Theorems of Uncertain Random Process
}

\author{
Kai $\mathrm{Yao}^{a}$, Jinwu $\mathrm{Gao}^{b}$ \\ ${ }^{a}$ Department of Mathematical Sciences, Tsinghua University, Beijing 100084, China \\ ${ }^{b}$ School of Information, Renmin University of China, Beijing 100872, China \\ yaok09@mails.tsinghua.edu.cn, jgao@ruc.edu.cn
}

\begin{abstract}
As a mixture of randomness and uncertainty, uncertain random variable is a measurable function from a probability space to a collection of uncertain variables. This paper will propose an uncertain random process as a generalization of both stochastic process and uncertain process. Special types of uncertain random process such as independent and stationary increment uncertain random process and uncertain random renewal process will also be discussed.
\end{abstract}

Keywords: uncertain random variable, uncertain process, stochastic process, uncertain random process

\section{$\S 1$ Introduction}

Probability theory has been used to model random phenomena for a long time. A premise of applying probability is that the obtained probability distribution is close enough to the real frequency. However, due to economic reasons or technical difficulties, we are often lack of observed data to estimate the distribution via statistics. In this case, some domain experts are invited to evaluate their belief degree of the possible events. However, the belief degree usually has a much larger range than the true frequency, and probability theory is not applicable by Liu [6]. In order to deal with this problem, an uncertainty theory was founded by Liu [1] in 2007 and refined by

Proceedings of the Twelfth International Conference on Electronic Business, Xi'an, China, October 12-16, 2012, 291-296.
Liu [5] in 2010 based on normality, duality, subadditivity and product axioms.

In order to model dynamic uncertain phenomena, a concept of uncertain process was proposed by Liu [2] in 2008. Following that, as an uncertain counterpart of Wiener process, canonical process was proposed by Liu [4] in 2009. It is a stationary independent increment uncertain process, and the increments are normal uncertain variables. In order to model the sudden jumps in an uncertain system, an uncertain renewal process was proposed by Liu [2] in 2008. After that, Liu [5] proposed uncertain renewal reward process, and applied it to an insurance model. In 2012, Yao and Li [9] proposed uncertain alternating renewal process.

Sometimes, randomness and uncertainty exist simultaneously in a complex system. In order to model such a system, Liu [7] proposed uncertain random variable and derived its chance measure in 2012. Meanwhile, Liu [7] defined expected value and variance for uncertain random variable. Following that, Liu [8] presented the operational law of uncertain random variable, and proposed uncertain random programming as a branch of mathematical programming involving uncertain random variables.

In this paper, we will propose uncertain random process. The rest of this paper is structured as follows. The next section is intended to introduce some concepts of uncertain random variable. Then uncertain random process is proposed in Section 3. As a special type of uncertain random process, stationary 
increment uncertain random process is proposed in Section 4, and law of large numbers for uncertain random variables is proved. After that, a concept of uncertain random renewal process is given in Section 5. At last, some remarks are given in Section 6 .

\section{$\S 2 \quad$ Preliminary}

Uncertainty theory is a branch of axiomatic mathematics to deal with human uncertainty. In order to model a system with both randomness and uncertainty, a chance theory was founded by Liu $[7,8]$ in 2012. In this section, we will introduce some useful definitions about uncertain variable and uncertain random variable.

\section{$\S 2.1 \quad$ Uncertain Variable}

Definition 1 (Liu [1]) Let $\mathcal{L}$ be a $\sigma$-algebra on a nonempty set $\Gamma$. A set function $\mathcal{M}: \mathcal{L} \rightarrow[0,1]$ is called an uncertain measure if it satisfies the following axioms:

Axiom 1: (Normality Axiom) $\mathcal{M}\{\Gamma\}=1$ for the universal set $\Gamma$.

Axiom 2: (Duality Axiom) $\mathcal{M}\{\Lambda\}+\mathcal{M}\left\{\Lambda^{c}\right\}=1$ for any event $\Lambda$.

Axiom 3: (Subadditivity Axiom) For every countable sequence of events $\Lambda_{1}, \Lambda_{2}, \cdots$, we have

$$
\mathcal{M}\left\{\bigcup_{i=1}^{\infty} \Lambda_{i}\right\} \leq \sum_{i=1}^{\infty} \mathcal{M}\left\{\Lambda_{i}\right\} .
$$

Besides, the product uncertain measure on the product $\sigma$-algebre $\mathcal{L}$ is defined by Liu [4] as follows,

Axiom 4: (Product Axiom) Let $\left(\Gamma_{k}, \mathcal{L}_{k}, \mathcal{M}_{k}\right)$ be uncertainty spaces for $k=1,2, \cdots$ Then the product uncertain measure $\mathcal{M}$ is an uncertain measure satisfying

$$
\mathcal{M}\left\{\prod_{i=1}^{\infty} \Lambda_{k}\right\}=\bigwedge_{k=1}^{\infty} \mathcal{M}_{k}\left\{\Lambda_{k}\right\}
$$

where $\Lambda_{k}$ are arbitrarily chosen events from $\mathcal{L}_{k}$ for $k=1,2, \cdots$, respectively.
An uncertain variable is essentially a measurable function from an uncertain space to the set of real numbers. In order to describe an uncertain variable, a concept of uncertainty distribution is defined as follows,

Definition 2 (Liu [1]) Let $\xi$ be an uncertain variable. Then its uncertainty distribution is defined by

$$
\Phi(x)=\mathcal{M}\{\xi \leq x\}
$$

for any real number $x$.

An uncertainty distribution $\Phi$ is said to be regular if its inverse function $\Phi^{-1}(\alpha)$ exists and is unique for each $\alpha \in(0,1)$. Inverse uncertainty distribution plays an important role in the operation of independent uncertain variables.

Definition 3 (Liu [4]) The uncertain variables $\xi_{1}, \xi_{2}, \cdots, \xi_{m}$ are said to be independent if

$$
\mathcal{M}\left\{\bigcap_{i=1}^{m}\left(\xi_{i} \in B_{i}\right)\right\}=\bigwedge_{k=1}^{m} \mathcal{M}\left\{\xi_{i} \in B_{i}\right\}
$$

for any Borel sets $B_{1}, B_{2}, \cdots, B_{m}$ of real numbers.

\section{§2.2 Uncertain Random Variable}

Definition 4 (Liu [7]) An uncertain random variable is a function $\xi$ from a probability space $(\Omega, \mathcal{A}, \operatorname{Pr})$ to the set of uncertain variables such that

$$
\mathcal{M}\{\xi(\omega) \in B\}
$$

is a measurable function of $\omega$ for any Borel set $B$ of 凡.

Random variables and uncertain variables can be regarded as special cases of uncertain random variables. Let $\eta$ be a random variable, $\tau$ be an uncertain variable. Then $\eta+\tau$ and $\eta \times \tau$ are both uncertain random variables.

Definition 5 (Liu [7]) Let $\xi$ be an uncertain random variable on a probability space $(\Omega, \mathcal{A}, \operatorname{Pr})$, and 
let $B$ be a Borel set of $\Re$. Then the chance of an uncertain random event $\xi \in B$ is defined by

$\operatorname{Ch}\{\xi \in B\}=\int_{0}^{1} \operatorname{Pr}\{\omega \in \Omega \mid \mathcal{M}\{\xi(\omega) \in B\} \geq r\} \mathrm{d} r$.

Chance measure for an uncertain random variable agrees with the probability measure for a random variable and uncertain measure for an uncertain variable, i.e., for any given Borel set $B$ of real numbers, we always have

$$
\operatorname{Ch}\{\eta \in B\}=\operatorname{Pr}\{\eta \in B\}
$$

for a random variable $\eta$ and

$$
\operatorname{Ch}\{\tau \in B\}=\mathcal{M}\{\tau \in B\}
$$

for an uncertain variable $\tau$. Besides, Liu [7] proved that a chance measure satisfies normality, duality, and monotonicity properties, that is

i) $\operatorname{Ch}\{\xi \in \Re\}=1$

ii) $\operatorname{Ch}\{\xi \in B\}+\operatorname{Ch}\left\{\xi \in B^{c}\right\}=1$ for any real number set $B$.

iii) $\operatorname{Ch}\left\{\xi \in B_{1}\right\} \leq \mathrm{Ch}\left\{\xi \in B_{2}\right\}$ for any real number set $B_{1} \subset B_{2}$.

Definition 6 (Liu [7]) Let $\xi$ be an uncertain random variable. Then its chance distribution of $\xi$ is defined by

$$
\Phi(x)=\operatorname{Ch}\{\xi \leq x\}
$$

for any $x \in \Re$.

The chance distribution of a random variable is just its probability distribution, and the chance distribution of an uncertain variable is just its uncertainty distribution.

Definition 7 (Liu [7]) Let $\xi$ be an uncertain random variable. Then its expected value is defined by

$$
E[\xi]=\int_{0}^{+\infty} \operatorname{Ch}\{\xi \geq r\} \mathrm{d} r-\int_{-\infty}^{0} \operatorname{Ch}\{\xi \leq r\} \mathrm{d} r
$$

provided that at least one of the two integrals is finite.

For a random variable $\eta$ and an uncertain variable $\tau$, Liu [8] proved that $E[\eta+\tau]=E[\eta]+E[\tau]$ and $E[\eta \times \tau]=E[\eta] \times E[\tau]$.

\section{$\S 3 \quad$ Uncertain Random Process}

This section aims at proposing an uncertain random process to model a dynamic uncertain random system. Generally speaking, an uncertain random process is in fact a sequence of uncertain random variables indexed by time or space.

Definition 8 An uncertain random process is a function $X_{t}$ from an index set $T$ to a collection of uncertain random variables, i.e., for each $t \in T$, the function $X_{t}$ is an uncertain random variable.

Remark 1 Since $\mathcal{M}\left\{X_{t}(\omega) \in B\right\}$ is a measurable function of $\omega$, the function $\mathcal{M}\left\{X_{t}(\cdot) \in B\right\}$ is a stochastic process.

Example 1 A stochastic process is a special type of uncertain random process, because any real value is a special uncertain variable.

Example 2 An uncertain process is a special type of uncertain random process, because for any given time, it is a function from a probability space to an uncertain variable.

Example 3 Let $Y_{t}$ be a stochastic process, and $Z_{t}$ be an uncertain process. If $f$ is a measurable function, then

$$
X_{t}=f\left(Y_{t}, Z_{t}\right)
$$

is an uncertain random process.

Definition 9 Assume $X_{t}$ is an uncertain random process on a chance space $(\Omega, \mathcal{A}, \operatorname{Pr}) \times(\Gamma, \mathcal{L}, \mathcal{M})$. Then for each fixed $\omega^{*} \in \Omega$ and $\gamma^{*} \in \Gamma$, the function $X_{t}\left(\omega^{*}, \gamma^{*}\right)$ is called a sample path of the uncertain random process of $X_{t}$.

Note that each sample path is a function of $t$. An uncertain random process can also be regarded as a function from a chance space to the collection of all sample paths. 
Definition 10 Assume $X_{t}$ is an uncertain random process and $z$ is a given level. Then the uncertain random variable

$$
\xi_{z}=\inf \left\{t \geq 0 \mid X_{t}=z\right\}
$$

is called the first hitting time that $X_{t}$ reaches the level $z$.

Theorem 1 Let $X_{t}$ be an uncertain random process and $z$ be a given level. Then the first hitting time $\xi_{z}$ that $X_{t}$ reaches the level $z$ has an uncertainty distribution

$$
\Phi(s)= \begin{cases}\operatorname{Ch}\left\{\sup _{0 \leq t \leq s} X_{t} \geq z\right\}, & \text { if } X_{0}<z \\ \operatorname{Ch}\left\{\inf _{0 \leq t \leq s} X_{t} \geq z\right\}, & \text { if } X_{0}>z .\end{cases}
$$

\section{$\S 4$ Stationary Increment Process}

Definition 11 An uncertain random process $X_{t}$ is said to have stationary increments if, for any given $t>0$, the increments $X_{t+s}-X_{s}$ are identically distributed uncertain random variables for all $s>0$.

Example 4 Wiener process $W_{t}$ is a stationary increment uncertain random process.

Example 5 Canonical process $C_{t}$ is a stationary increment uncertain random process.

Example 6 Let $\eta_{1}, \eta_{2}, \cdots$ be a sequence of iid random variables, and $\tau_{1}, \tau_{2}, \cdots$ be a sequence of iid uncertain variables. Assume $f$ is a measurable function, then

$$
X_{n}=f\left(\eta_{1}, \tau_{1}\right)+f\left(\eta_{2}, \tau_{2}\right)+\cdots+f\left(\eta_{n}, \tau_{n}\right)
$$

is a stationary increment uncertain random process.

Theorem 2 Let $X_{t}$ be a stationary increment uncertain random process. Then for any real number a and $b$, the uncertain process

$$
Y_{t}=a X_{t}+b
$$

is also a stationary increment uncertain random process.

\section{$\S 5$ Renewal Process}

Definition 12 Let $\xi_{1}, \xi_{2}, \cdots$ be a sequence of positive uncertain random variables. Define $S_{0}=0$ and $S_{n}=\xi_{1}+\xi_{2}+\cdots+\xi_{n}$ for $n \geq 1$. Then

$$
N_{t}=\max _{n \geq 0}\left\{n \mid S_{n} \leq t\right\}
$$

is called an uncertain random renewal process.

Example 7 A stochastic renewal process is an uncertain random renewal process, because a random variable is a special uncertain random variable.

Example 8 An uncertain renewal process is an uncertain random renewal process, because an uncertain variable is a special uncertain random variable.

Theorem 3 Let $\eta_{1}, \eta_{2}, \cdots$ be a sequence of iid random variables with a common uncertainty distribution $\Phi, \tau_{1}, \tau_{2}, \cdots$ be a sequence of iid uncertain variables, and $f(x, y)$ be a positive measurable function and monotone with respect to $y$. Assume that $N_{t}$ is an uncertain random renewal process with interarrival times $f\left(\xi_{1}, \eta_{1}\right), f\left(\xi_{2}, \eta_{2}\right), \cdots$ Then

$$
\frac{N_{t}}{t} \rightarrow\left(\int_{-\infty}^{+\infty} f\left(x, \tau_{1}\right) \mathrm{d} \Phi(x)\right)^{-1}
$$

in the sense of convergence in distribution.

Proof: It follows from the definition of renewal process that

$$
\begin{aligned}
\operatorname{Ch}\left\{\frac{N_{t}}{t} \leq y\right\} & =\operatorname{Ch}\left\{N_{t} \leq t y\right\}=\operatorname{Ch}\left\{N_{t} \leq\lfloor t y\rfloor\right\} \\
& =\operatorname{Ch}\left\{S_{\lfloor t y\rfloor+1} \geq t\right\} \\
& =\operatorname{Ch}\left\{\frac{S_{\lfloor t y+1}}{\lfloor t y\rfloor+1} \geq \frac{t}{\lfloor t y\rfloor+1}\right\}
\end{aligned}
$$

for any $y \in(0,1)$ where $\lfloor t y\rfloor$ represents the maximal integer less than or equal to $t y$. It follows from the 
law of large numbers that

$$
\begin{aligned}
& \lim _{t \rightarrow+\infty} \operatorname{Ch}\left\{\frac{N_{t}}{t} \leq y\right\} \\
= & \mathcal{M}\left\{\int_{-\infty}^{+\infty} f\left(x, \tau_{1}\right) \mathrm{d} \Phi(x) \geq \frac{1}{y}\right\} \\
= & \mathcal{M}\left\{\left(\int_{-\infty}^{+\infty} f\left(x, \tau_{1}\right) \mathrm{d} \Phi(x)\right)^{-1} \leq y\right\}
\end{aligned}
$$

for almost all $y \in(0,1)$. Thus $N_{t} / t$ converges in distribution to

$$
\left(\int_{-\infty}^{+\infty} f\left(x, \tau_{1}\right) \mathrm{d} \Phi(x)\right)^{-1} .
$$

\section{$§ 5.1$ Renewal Reward Process}

Definition 13 Let $\xi_{1}, \xi_{2}, \cdots$ be uncertain random interarrival times, and $\zeta_{1}, \zeta_{2}, \cdots$ be uncertain random rewards. Then

$$
R_{t}=\sum_{i=1}^{N_{t}} \zeta_{i}
$$

is called an uncertain random renewal reward process, where $N_{t}$ is a renewal process with uncertain random interarrival times $\xi_{1}, \xi_{2}, \cdots$

Theorem 4 Let $N_{t}$ be a renewal process with iid random interarrival times $\eta_{1}, \eta_{2}, \cdots$ and let $\tau_{1}, \tau_{2}, \cdots$ be iid uncertain rewards. Then

$$
R_{t}=\sum_{i=1}^{N_{t}} \tau_{i}
$$

is an uncertain random renewal reward process, and

$$
\frac{R_{t}}{t} \rightarrow \frac{\tau_{1}}{E\left[\eta_{1}\right]}
$$

in the sense of convergence in distribution.

Proof: Let $\Phi$ denote the uncertainty distribution of $\eta_{1}$. It follows from the definition of chance distribu- tion that

$$
\begin{aligned}
& \operatorname{Ch}\left\{\frac{R_{t}}{t} \leq x\right\} \\
= & \int_{0}^{1} \operatorname{Pr}\left\{\mathcal{M}\left\{\frac{R_{t}}{t} \leq x\right\} \geq r\right\} \mathrm{d} r \\
= & \int_{0}^{1} \operatorname{Pr}\left\{\mathcal{M}\left\{\left(\sum_{i=1}^{N_{t}} \tau_{i} / N_{t}\right) \frac{N_{t}}{t} \leq x\right\} \geq r\right\} \mathrm{d} r \\
= & \int_{0}^{1} \operatorname{Pr}\left\{\mathcal{M}\left\{\left(\sum_{i=1}^{N_{t}} \tau_{i} / N_{t}\right) \leq \frac{t x}{N_{t}}\right\} \geq r\right\} \mathrm{d} r \\
= & \int_{0}^{1} \operatorname{Pr}\left\{\Phi\left(\frac{t x}{N_{t}}\right) \geq r\right\} \mathrm{d} r .
\end{aligned}
$$

Since $N_{t}$ is a random renewal process with iid interarrival times $\eta_{1}, \eta_{2}, \cdots$, we have

$$
\frac{t}{N_{t}} \rightarrow E\left[\eta_{1}\right] \quad \text { a.s. }
$$

It follows from the Lebesgue dominant convergence theorem that

$$
\begin{aligned}
& \lim _{t \rightarrow+\infty} \operatorname{Ch}\left\{\frac{R_{t}}{t} \leq x\right\} \\
= & \int_{0}^{1} \lim _{t \rightarrow+\infty} \operatorname{Pr}\left\{\Phi\left(\frac{t x}{N_{t}}\right) \geq r\right\} \mathrm{d} r \\
= & \int_{0}^{1} \operatorname{Pr}\left\{\Phi\left(E\left[\eta_{1}\right] x\right) \geq r\right\} \mathrm{d} r \\
= & \Phi\left(E\left[\eta_{1}\right] x\right)
\end{aligned}
$$

which is just the uncertainty distribution of $\tau_{1} / E\left[\eta_{1}\right]$ The theorem is thus verified.

\section{$\S 6$ Conclusion}

This paper first proposed a concept of uncertain random process. As a special type of uncertain random process, this paper also proposed stationary increment uncertain random process. In addition, this paper studied uncertain random renewal process, and proved the fundamental renewal theorem.

\section{Acknowledgements}

This work was supported by National Natural Science Foundation of China Grant No.61273044. 


\section{References}

[1] Liu B., Uncertainty Theory, 2nd ed., SpringerVerlag, Berlin, 2007.

[2] Liu B., Fuzzy process, hybrid process and uncertain process, Journal of Uncertain Systems, Vol.2, No.1, 3-16, 2008.

[3] Liu B., Theory and Practice of Uncertain Programming, 2nd ed., Springer-Verlag, Berlin, 2009.

[4] Liu B., Some research problems in uncertainty theory, Journal of Uncertain Systems, Vol.3, No.1, 3-10, 2009.

[5] Liu B., Uncertainty Theory: A Branch of Mathematics for Modeling Human Uncertainty, Springer-Verlag, Berlin, 2010.
[6] Liu B., Why is there a need for uncertainty theory? Journal of Uncertain Systems, Vol.6, No.1, 3-10, 2012.

[7] Liu Y.H., Uncertain random variables: A mixture of uncertainty and randomness, Soft Computing, to be published.

[8] Liu Y.H., Uncertain random programming with applications, Fuzzy Optimization and Decision Making, to be published.

[9] Yao K., and Li X., Uncertain alternating renewal process and its application, IEEE Transactions on Fuzzy Systems, to be published.

[10] Zhu Y., Uncertain optimal control with application to a portfolio selection model, Cybernetics and Systems, Vol.41, No.7, 535-547, 2010. 